\title{
Calkin algebras for Banach spaces with finitely decomposable quotients
}

\author{
by \\ Manuel González and José M. Herrera (Santander)
}

\begin{abstract}
For a Banach space $X$ such that all quotients only admit direct decompositions with a number of summands smaller than or equal to $n$, we show that every operator $T$ on $X$ can be identified with an $n \times n$ scalar matrix modulo the strictly cosingular operators $S C(X)$. More precisely, we obtain an algebra isomorphism from the Calkin algebra $L(X) / S C(X)$ onto a subalgebra of the algebra of $n \times n$ scalar matrices which is triangularizable when $X$ is indecomposable. From this fact we get some information on the class of all semi-Fredholm operators on $X$ and on the essential spectrum of an operator acting on $X$.
\end{abstract}

1. Introduction. A Banach space is indecomposable if each complemented subspace of $X$ is finite-dimensional or finite-codimensional. Gowers and Maurey [7] constructed a Banach space $X_{\mathrm{GM}}$ which is hereditarily indecomposable, i.e., every subspace of $X_{\mathrm{GM}}$ is indecomposable. This example gives a negative answer to several long-standing open problems; for example, $X_{\mathrm{GM}}$ contains no unconditional basic sequences. We refer to $[1,8]$ for other examples of hereditarily indecomposable spaces. We say that a Banach space is $n$-decomposable if it can be written as a direct sum of $n$ infinite-dimensional closed subspaces.

Here we study the structure of the Calkin algebra $L(X) / S C(X)$, where $S C(X)$ stands for the strictly cosingular operators on an (infinite-dimensional) Banach space $X$ which has $n$-decomposable quotients but has no $(n+1)$-decomposable quotients. We denote by $Q D_{n}$ the class of such spaces and we call the class $Q D_{1}$ the quotient indecomposable spaces. The space $X_{\mathrm{GM}}$ is quotient indecomposable [3]. If the dual space $X^{*}$ is hereditarily decomposable, then $X$ is quotient indecomposable. Moreover, the product of $n$ copies of $Q D_{1}$ spaces is a $Q D_{n}$ space [6, Theorem 1]. However, these examples do not exhaust the class $Q D_{n}$ (see Remark 3.3(c)).

2000 Mathematics Subject Classification: Primary 47A10, 47A53; Secondary 46B20.

Key words and phrases: quotient indecomposable Banach spaces, strictly cosingular operators, essential spectrum, Fredholm operators, Calkin algebras.

Supported in part by DGI (Spain), Grant BFM2001-1147. 
We show that for each complex space $X \in Q D_{n}$, there exists an algebra isomorphism from $L(X) / S C(X)$ onto a subalgebra of the algebra $M_{n}(\mathbb{C})$ of $n \times n$ scalar matrices. Since the class $\Phi(X)$ of Fredholm operators on $X$ coincides with the class of those $T \in L(X)$ such that the corresponding class in $L(X) / S C(X)$ is invertible, we identify $\Phi(X)$ with the set of all invertible elements of a subalgebra of $M_{n}(\mathbb{C})$ and we prove that the essential spectrum of an operator $T \in L(X)$ coincides with the spectrum of an $n \times n$ matrix.

We also show that a complex Banach space is $n$-decomposable if and only if there is an operator $T$ on it whose essential spectrum admits a partition into $n$ non-empty compact subsets. Applying this result to the case where $X$ is an indecomposable $Q D_{n}$ space, we deduce that the Calkin algebra $L(X) / S C(X)$ can be identified with a subalgebra of the algebra of all upper triangular $n \times n$ matrices with constant diagonal.

For this purpose, we study the quotient spaces $L(X, Y) / S C(X, Y)$ when $X \in Q D_{n}$ and $Y \in Q D_{m}$. First we consider the case in which $X$ and $Y$ are products of $Q D_{1}$ spaces. Then we apply the results to the general case. Whenever $Y \in Q D_{m}$ is isomorphic to a quotient of $X \in Q D_{n}$ we show that $L(X, Y) / S C(X, Y)$ can be identified with a subspace of the space of $m \times n$ scalar matrices. From this result we obtain a representation of the Calkin algebra $L(X) / S C(X)$ for $X$ a $Q D_{n}$ space.

Some of the results and ideas behind this paper are a dual version of the results of Ferenczi [2], who considers hereditarily indecomposable spaces and strictly singular operators. However, our development is more closely related to operator theory. In this way our proofs are shorter and our presentation is more transparent. Moreover, we obtain a representation of $L(X) / S C(X)$ as an algebra of matrices, which gives additional information on the Calkin algebra. We observe that our scheme could be applied to the case considered in [2], improving in this way the results contained there.

Along the paper $X, Y, Z, \ldots$ will denote Banach spaces over the field of real or complex numbers. All statements are valid in both cases unless explicitly mentioned otherwise. $X^{*}$ will stand for the dual space of $X$ and $L(X, Y)$ for the (continuous linear) operators from $X$ into $Y$. We set $L(X)=$ $L(X, X)$, and $I$ is the identity map. Given operators $S \in L(Z, X)$ and $T \in L(Z, Y)$, we define their product $S \times T \in L(Z, X \times Y)$ by $(S \times T)(z):=$ $(S(z), T(z))$.

Subspaces will always be closed and infinite-dimensional, and quotients will be infinite-dimensional. We denote the quotient map from a Banach space $X$ onto a quotient $Y$ by $Q_{Y}$.

Given subsets $A \subseteq X, B \subseteq X^{*}$, we denote by $A^{\perp},{ }_{\perp} B$, their respective annihilators in $X^{*}$ and $X$. 
2. Quasi-maximal quotients. In this section we describe the quasimaximal quotients of a Banach space. We also give some results that we will need later.

Definition 2.1. We say that a quotient $Y$ of $X$ is quasi-maximal if there is no quotient $Z$ of $X$ such that $Q_{Y} \times Q_{Z}$ is surjective.

We say that the space $X$ is quotient indecomposable if no quotient of $X$ can be written as the direct sum of two subspaces.

Clearly, $X$ is quotient indecomposable if and only if every quotient of $X$ is quasi-maximal.

Definition 2.2. An operator $T \in L(X, Y)$ is said to be strictly cosingular, $T \in S C(X, Y)$, if there is no quotient $Z$ of $Y$ so that $Q_{Z} T$ is surjective.

An operator $T \in L(X, Y)$ is said to be lower semi-Fredholm, $T \in$ $\Phi_{-}(X, Y)$, if $R(T)$ is closed and $Y / R(T)$ is finite-dimensional.

The following two results will be useful.

Proposition 2.3 [5, Corollary 1]. Suppose that $Y$ is quotient indecomposable. Then for every $X$,

$$
L(X, Y)=\Phi_{-}(X, Y) \cup S C(X, Y) .
$$

Lemma 2.4. Let $M$ and $N$ be subspaces of $X$. Suppose that $M+N$ is infinite-codimensional. Let $0<\varepsilon<1$. Then there exists a compact operator $K \in L(X)$ with $\|K\|<\varepsilon$ such that

(a) $I+K$ induces an isomorphism from a quotient of $X / N$ onto a quotient of $X / M$,

(b) $I+K^{*}$ induces an isomorphism from a weak ${ }^{*}$ closed subspace of $M^{\perp}$ into $N^{\perp}$.

Proof. If $M+N$ is closed, then $(M+N)^{\perp}$ is a weak* closed subspace contained in both $M^{\perp}$ and $N^{\perp}$, and $X /(M+N)$ is a quotient of both $X / M$ and $X / N$. Thus, we get the result by setting $K=0$.

Suppose that $M+N$ is not closed; then $M^{\perp}+N^{\perp}$ is also not closed [10, Theorem IV.4.8]. In particular, $M^{\perp}+N^{\perp}$ is not a direct sum. Thus

$$
\inf \left\{\|f-g\|: f \in M^{\perp}, g \in N^{\perp},\|f\|=\|g\|=1\right\}=0 .
$$

By (1) we can find $f_{1} \in M^{\perp}, g_{1} \in N^{\perp}$ such that $\left\|f_{1}\right\|=\left\|g_{1}\right\|=1$ and $\left\|f_{1}-g_{1}\right\|<\varepsilon / 2^{2}$. Then we choose $x_{1} \in X$ such that $\left\|x_{1}\right\|<2$ and $f_{1}\left(x_{1}\right)=1$.

Assume that we have selected $f_{i} \in M^{\perp}, g_{i} \in N^{\perp}$ and $x_{i} \in X$ such that

$$
\left\|f_{i}\right\|=\left\|g_{i}\right\|=1, \quad f_{i}\left(x_{j}\right)=\delta_{i j}, \quad\left\|x_{i}\right\|\left\|f_{i}-g_{i}\right\|<\frac{\varepsilon}{2^{i}}
$$

for $i, j \leq n$. We set $F_{n}=\operatorname{span}\left\{x_{1}, \ldots, x_{n}\right\}$ and $G_{n}=\operatorname{span}\left\{f_{1}, \ldots, f_{n}\right\}$. Then

$$
X=F_{n} \oplus{ }_{\perp} G_{n}, \quad X^{*}=F_{n}^{\perp} \oplus G_{n} .
$$


Let $P_{n}$ be the projection on $X$ with $\operatorname{Ker} P_{n}=F_{n}$ and $R\left(P_{n}\right)={ }_{\perp} G_{n}$. Note that $\left(M+F_{n}\right)+N$ is not closed. Again by (1) we can choose $f_{n+1} \in\left(M+F_{n}\right)^{\perp}$ and $g_{n+1} \in N^{\perp}$ such that $\left\|f_{n+1}\right\|=\left\|g_{n+1}\right\|=1$ and

$$
\left\|f_{n+1}-g_{n+1}\right\|<\frac{\varepsilon}{2^{n+2}\left\|P_{n}\right\|} \text {. }
$$

We take $y_{n+1} \in X$ such that $\left\|y_{n+1}\right\|<2$ and $f_{n+1}\left(y_{n+1}\right)=1$, and set $x_{n+1}=P_{n}\left(y_{n+1}\right)$. Clearly $f_{n+1}\left(x_{i}\right)=\delta_{n+1, i}$ for $i=1, \ldots, n+1$. Moreover,

$$
\left\|x_{n+1}\right\|\left\|f_{n+1}-g_{n+1}\right\|<\frac{\varepsilon}{2^{n+1}} .
$$

We define an operator $K: X \rightarrow X$ by $K(x)=\sum_{n=1}^{\infty}\left(g_{n}-f_{n}\right)(x) x_{n}$. Note that $\|K\| \leq \sum_{n=1}^{\infty}\left\|g_{n}-f_{n}\right\|\left\|x_{n}\right\|<\varepsilon$. Therefore $I+K$ is an isomorphism on $X$.

Set $M_{0}=\bigcap_{n=1}^{\infty} \operatorname{Ker} f_{n}$ and $N_{0}=\bigcap_{n=1}^{\infty} \operatorname{Ker} g_{n}$. Then we have $M \subseteq M_{0}$ and $N \subseteq N_{0}$, so $M_{0}^{\perp} \subseteq M^{\perp}$ and $N_{0}^{\perp} \subseteq N^{\perp}$. It is not difficult to check that $(I+K)^{*} f_{n}=g_{n}$, whence $\operatorname{Ker} f_{n}=(I+K)\left(\operatorname{Ker} g_{n}\right)$. Since $I+K$ is bijective, $(I+K)\left(\bigcap_{n=1}^{\infty} \operatorname{Ker} g_{n}\right)=\bigcap_{n=1}^{\infty}(I+K)\left(\operatorname{Ker} g_{n}\right)$ and $(I+K) N_{0}=M_{0}$. Therefore $I+K$ induces an isomorphism from $X / N_{0}$ onto $X / M_{0}$, so part (a) follows. Moreover $(I+K)^{*} M_{0}^{\perp}=N_{0}^{\perp}$ and part (b) follows as well.

Definition 2.5. Let $Y$ and $Z$ be quotients of $X$. We say that $Y$ and $Z$ are $(I+K)$-isomorphic if there is an operator $K: X \rightarrow X$ such that $I+K$ induces an isomorphism from $Y$ onto $Z$.

Proposition 2.6. Let $Y$ and $Z$ be quotients of $X$ and let $\varepsilon>0$. Suppose that there is no quotient $W$ of $Z$ such that the map $Q_{Y} \times Q_{W}$ is surjective. Then $Y$ and $Z$ have $(I+K)$-isomorphic quotients with $\|K\|<\varepsilon$. This is the case, in particular, when $Y$ is quasi-maximal.

Proof. Let $Y=X / M$ and $Z=X / N$. By the hypothesis, for any infinitecodimensional subspace $L$ of $X$ such that $L \supseteq N$, we have $M+L \neq X$. Thus $M+N$ is infinite-codimensional and Lemma 2.4 applies.

Corollary 2.7. Let $X$ be a quotient indecomposable space and let $Y$ and $Z$ be quotients of $X$. Then $Y$ has a quotient isomorphic to a quotient of $Z$.

Proposition 2.8. Let $Y$ be a quasi-maximal quotient of $X$.

(a) An operator $S \in L(Z, X)$ is strictly cosingular if and only if $Q_{Y} S$ is. Therefore

$$
\operatorname{dim} L(Z, X) / S C(Z, X) \leq \operatorname{dim} L(Z, Y) / S C(Z, Y) .
$$

(b) For each $T \in L(X, Z)$ there is a quotient $Z_{1}$ of $Z$ and $T_{1}: Y \rightarrow Z_{1}$ such that $Q_{Z_{1}} T-T_{1} Q_{Y}$ is strictly cosingular. 
Proof. (a) If $S: Z \rightarrow X$ is not strictly cosingular, then we have a quotient $X_{1}$ of $X$ such that $Q_{X_{1}} S$ is surjective. As $Y$ is a quasi-maximal quotient, Proposition 2.6 applies so that there exist $K \in L(X)$ with $\|K\|<1 / 2$ and $(I+K)$-isomorphic quotients $Y_{1}$ of $Y$ and $Z_{1}$ of $X_{1}$. Let $U \in L\left(Y_{1}, Z_{1}\right)$ be the isomorphism induced by $I+K$ and let $Q$ denote the quotient map from $Y$ onto $Y_{1}$. Since $\|K\|<1 / 2, I+K$ is an isomorphism. Thus $Q Q_{Y} S=$ $U^{-1} Q_{Z_{1}}(I+K) S$ is surjective and $Q_{Y} S$ is not strictly cosingular. For the second part, it is enough to observe that the correspondence $[S] \mapsto\left[Q_{Y} S\right]$ defines a one-to-one map from $L(Z, X) / S C(Z, X)$ into $L(Z, Y) / S C(Z, Y)$.

(b) Let $T \in L(X, Z)$. If $T$ is strictly cosingular, then we take $Z_{1}=Z$ and $T_{1}=0$. Suppose that $T$ is not strictly cosingular. Then there is a quotient $W$ of $Z$ such that $Q_{W} T$ is surjective. Now $Y$ is a quasi-maximal quotient, so Proposition 2.6 applies to $Q_{W} T$ and $Q_{Y}$, and we get quotients $Y_{1}$ of $Y$ and $Z_{1}$ of $W$, a compact operator $K: X \rightarrow X$ and an isomorphism $\phi: Y_{1} \rightarrow Z_{1}$ so that $Q_{Z_{1}} T(I+K)=\phi Q_{Y_{1}}$. Let $q$ denote the quotient map from $Y$ onto $Y_{1}$. Set $T_{1}=\phi q$. Then $Q_{Z_{1}} T-T_{1} Q_{Y}=-Q_{Z_{1}} T K \in S C\left(X, Z_{1}\right)$.

3. Operators on fundamental $Q D_{n}$ spaces. Recall that $X$ and $Y$ are quotient incomparable if no quotient of $X$ is isomorphic to a quotient of $Y$, and $X$ is said to be $n$-decomposable if $X=X_{1} \oplus \ldots \oplus X_{n}$, with $X_{1}, \ldots, X_{n}$ subspaces of $X$.

Definition 3.1. We say that a Banach space $X$ is a $Q D_{n}$ space, $X \in Q D_{n}$, if $X$ has an $n$-decomposable quotient, but it has no $(n+1)$ decomposable quotient.

We say that $X$ is quotient indecomposable if it is a $Q D_{1}$ space.

Definition 3.2. A $Q D_{n}$ space $X$ is fundamental if it is a product $X=$ $\prod_{i=1}^{n} X_{i}$ of quotient indecomposable spaces such that, for every $i, j=1, \ldots, n$, either $X_{i}=X_{j}$, or $X_{i}, X_{j}$ are quotient incomparable.

For a fundamental $Q D_{n}$ space $X=\prod_{i=1}^{n} X_{i}$ including exactly $k$ different factors, we define the characteristic of $X$ as

$$
\chi(X):=\left(n_{1}, \ldots, n_{k}\right),
$$

where $n_{1} \leq \ldots \leq n_{k}$ are the times that each space appears in the product, arranged in increasing order.

REMARK 3.3. (a) The definition of a fundamental $Q D_{n}$ space looks very restrictive, since we require some spaces to be equal, not just isomorphic. However, it will allow us to simplify some arguments.

(b) It follows readily from the definition that $X \in Q D_{n}$ if and only if it has a quasi-maximal quotient isomorphic to a fundamental $Q D_{n}$ space.

(c) The product of $n$ quotient indecomposable spaces is a $Q D_{n}$ space [6, Theorem 1]. However, these products do not exhaust the class of all $Q D_{n}$ 
spaces: for each $n$ there exists a hereditarily indecomposable space $\widehat{X}$ which is a $Q D_{n}$ space.

Indeed, it was proved by Ferenczi [3, Appendix] that for each $n \in \mathbb{N}$, there exist complex spaces $X_{1}, \ldots, X_{n}$ such that every quotient of each $X_{i}$ is hereditarily indecomposable, and a subspace $Z$ of $X_{1} \times \ldots \times X_{n}$ so that

$$
\widehat{X}:=\frac{X_{1} \times \ldots \times X_{n}}{Z}
$$

is hereditarily indecomposable. Moreover, $\widehat{X}$ has a quotient which is $n$ decomposable. Thus $\widehat{X}$ is a $Q D_{n}$ space.

Let $X=\prod_{j=1}^{n} X_{j} \in Q D_{n}$ and $Y=\prod_{i=1}^{m} Y_{i} \in Q D_{m}$ be fundamental spaces and let $\Psi: X \rightarrow Y$ be an operator. We will identify $\Psi$ with the $m \times n$ matrix $\left(\Psi_{i j}\right)$, where $\Psi_{i j}: X_{j} \rightarrow Y_{i}$ is the $i$ th coordinate of the restriction of $\Psi$ to $X_{j}$. The operator $\Psi$ is strictly cosingular if and only if every $\Psi_{i j}$ is strictly cosingular; in this case we will say that $\left(\Psi_{i j}\right)$ is a strictly cosingular matrix. In the case $n=m$, we say that $\Psi$ is diagonal when $\Psi_{i j}=0$ for $i \neq j$.

We denote by $M_{X, Y}$ the set of all $m \times n$ scalar matrices $A=\left(a_{i j}\right) \in M_{m, n}$ such that $a_{i j}=0$ when $X_{j}$ and $Y_{i}$ are quotient incomparable. Sometimes we will write $M_{m, n}(\mathbb{C})$ to emphasize that we are considering the field of complex numbers.

If $\chi(X)=\left(n_{1}, \ldots, n_{k}\right)$, then it is clear that

$$
\operatorname{dim} M_{X, X}=n_{1}^{2}+\ldots+n_{k}^{2} .
$$

Given a product space $X=\prod_{j=1}^{n} X_{j}$ and a permutation $\sigma$ of $\{1, \ldots, n\}$ such that $X_{\sigma(i)}=X_{i}$ for every $i$, we denote by $V_{\sigma}$ the operator defined by $V_{\sigma}\left(x_{1}, \ldots, x_{n}\right):=\left(x_{\sigma(1)}, \ldots, x_{\sigma(n)}\right)$, and we say that $V_{\sigma}$ is an allowable permutation of the factors of $X$.

If $A \in M_{m, p}$ we denote by $(A \mid 0) \in M_{m, p+q}$ the matrix whose first $p$ columns are those of $A$ and the rest of the entries are 0.

Proposition 3.4. Let $X=\prod_{j=1}^{n} X_{j} \in Q D_{n}$ and $Y=\prod_{i=1}^{m} Y_{i} \in Q D_{m}$ be fundamental spaces. Suppose that $Y$ is isomorphic to a quotient of $X$. Then there exists a quotient $Z$ of $Y$ which is a fundamental $Q D_{m}$ space and a surjective operator from $X$ onto $Z$ with matrix $(D \mid 0) V_{\sigma}$, where $D$ is an $m \times m$ diagonal surjective matrix and $V_{\sigma}$ is an allowable permutation of the factors of $X$.

Proof. Let $\Psi=\left(\Psi_{i j}\right): X \rightarrow Y$ be a surjective operator, where $\Psi_{i j} \in$ $L\left(X_{j}, Y_{i}\right)$. Clearly, for each $i$ there is at least one $j$ so that $\Psi_{i j}$ is not strictly cosingular. Hence $\Psi_{i j}$ is lower semi-Fredholm, by Proposition 2.3. Thus, if we pass to a finite-codimensional subspace of $Y_{i}$, which we also denote by $Y_{i}$, then there exists a surjective map $q_{i}: X_{j} \rightarrow Y_{i}$. We take as $Z$ the product of these new spaces $Y_{i}$. 
Suppose that $\Psi_{i j}$ and $\Psi_{k l}$ are not strictly cosingular. Then the definition of a fundamental $Q D_{n}$ space and Corollary 2.7 imply that

$$
Y_{i}=Y_{k} \quad \text { if and only if } \quad X_{j}=X_{l} .
$$

Since the sum of a lower semi-Fredholm operator and a strictly cosingular operator is lower semi-Fredholm [11, Chap. V, Theorem 3.4], the operator from the product of copies of $X_{j}$ into the product of copies of $Y_{i}$ induced by $\Psi$ is lower semi-Fredholm. Thus, taking into account that the product of $k$ quotient indecomposable spaces is a $Q D_{k}$ space [6, Theorem 1], we conclude that the number of copies of $Y_{i}$ in $Y$ is smaller than or equal to the number of copies of $X_{j}$ in $X$.

It is enough to select a suitable $V_{\sigma}$ and take as $D$ a diagonal matrix with a number of copies of $q_{i}$ on the diagonal as necessary.

REMARK 3.5. Similarly to [2, Lemma 5], we could have proved a result stronger than Proposition 3.4. Namely, for each couple of fundamental spaces $X \in Q D_{n}, Y \in Q D_{m}$ and each surjective operator $\Psi: X \rightarrow Y$, there exist a quotient $Z$ of $Y$ which is a fundamental $Q D_{m}$ space, an automorphism $U$ of $Z$ and a permutation $V_{\sigma}$ of the factors of $X$ so that

$$
U Q_{Z} \Psi V_{\sigma}=(D+K \mid B),
$$

where $D$ is a diagonal surjective $m \times m$ matrix, $K$ is a strictly cosingular $m \times m$ matrix and $B$ is an $m \times(n-m)$ matrix.

However, we do not need this result in its full generality.

Remark 3.6. Let $Y=\prod_{i=1}^{m} Y_{i} \in Q D_{m}$ and $X=\prod_{j=1}^{n} X_{j} \in Q D_{n}$ be fundamental spaces. Suppose that there exists a surjective operator from $X$ onto $Y$ determined by a matrix $(D \mid 0)$, where $D=\operatorname{diag}\left(q_{1}, \ldots, q_{m}\right)$ is an $m \times m$ diagonal matrix. Let $A \in M_{X, Y}$. Then $a_{i j}=0$ whenever $X_{i} \neq X_{j}$. We will denote by $D A$ the operator from $X$ into $Y$ with entries $a_{i j} q_{i}$.

Moreover, if $\chi(X)=\left(n_{1}, \ldots, n_{k}\right)$ and $\chi(Y)=\left(m_{1}, \ldots, m_{l}\right)$, then

$$
\operatorname{dim} M_{X, Y}=m_{1} n_{1}+\ldots+m_{l} n_{l} .
$$

The following result was essentially proved in [5]:

Proposition 3.7. Let $Z$ be a complex quotient indecomposable space. Then for every surjective operator $q: Z \rightarrow X$ we can write

$$
L(Z, X)=\mathbb{C} q \oplus S C(Z, X) .
$$

Proof. Observe that there exists an isomorphism $\Psi$ from a quotient $Y$ of $Z$ onto $X$ so that $q=\Psi Q_{Y}$. By [5, Theorem 4], $L(Z, Y)=\mathbb{C} Q_{Y} \oplus S C(Z, Y)$. Let $T \in L(Z, X)$. Then $\Psi^{-1} T=\lambda Q_{Y}+K$ for some $\lambda \in \mathbb{C}$ and some $K \in S C(Z, Y)$. Thus $T=\lambda q+\Psi K$ with $\Psi K \in S C(Z, Y)$.

Proposition 3.8. Let $X \in Q D_{n}$ and $Y \in Q D_{m}$ be complex fundamental spaces. Suppose that there exists a surjective operator from $X$ onto $Y$ with 
matrix $(D \mid 0)$, where $D$ is an $m \times m$ diagonal matrix. Then for every $T \in$ $L(X, Y)$ there exist a matrix $A \in M_{X, Y}(\mathbb{C})$ and an operator $K \in S C(X, Y)$ such that

$$
T=D A+K
$$

Proof. Let $\left(T_{i j}\right)$ be the matrix associated to $T$. Set $D=\operatorname{diag}\left(q_{1}, \ldots, q_{m}\right)$. Then by Proposition 3.7, $T_{i j}=a_{i j} q_{i}+K_{i j}$, where $a_{i j} \in \mathbb{C}$ and $K_{i j} \in$ $S C\left(X_{j}, Y_{i}\right)$. Moreover, by Remark 3.6, $a_{i j}=0$ for $X_{i} \neq X_{j}$. In matrix terms, this is equivalent to the equality

$$
\left(T_{i j}\right)=\operatorname{diag}\left(q_{1}, \ldots, q_{m}\right)\left(a_{i j}\right)+\left(K_{i j}\right)
$$

where $A=\left(a_{i j}\right) \in M_{X, Y}(\mathbb{C})$ and $K=\left(K_{i j}\right) \in S C(X, Y)$.

4. Operators on $Q D_{n}$ spaces. Here we show that some properties of the operators on a $Q D_{n}$ space $X$ can be derived from those obtained for a fundamental $Q D_{n}$ space. The key will be Theorem 4.8, in which we use a filter defined in the class of all fundamental quotients of $X$ (see Definition 4.1 below) to identify matrices and operators, modulo the strictly cosingular operators.

Definition 4.1. Let $X \in Q D_{n}$. A fundamental quotient of $X$ is a pair $(\widetilde{X}, U)$, where $\widetilde{X}$ is a quotient of $X$ and $U$ is an isomorphism from $\widetilde{X}$ onto a fundamental $Q D_{n}$ space $\prod_{i=1}^{n} X_{i}$. We usually write $\widetilde{X} \simeq \prod_{i=1}^{n} X_{i}$ or simply $\widetilde{X}$.

Let $\widetilde{X}_{1} \simeq \prod_{i=1}^{n} X_{i}$ and $\widetilde{X}_{2} \simeq \prod_{i=1}^{n} Y_{i}$ be two fundamental quotients of $X$. We say that $\widetilde{X}_{1}$ is a diagonal quotient of $\widetilde{X}_{2}$ if there exists a diagonal surjective matrix from $\prod_{i=1}^{n} Y_{i}$ onto $\prod_{i=1}^{n} X_{i}$.

We write $\widetilde{X}_{1} \leq \widetilde{X}_{2}$ when $\prod_{i=1}^{n} X_{i}$ is a diagonal quotient of $\prod_{i=1}^{n} Y_{i}$ up to a permutation of the factors.

REMARK 4.2. Every fundamental quotient $(\widetilde{X}, U)$ of $X$ is quasi-maximal.

Proposition 4.3. Every $X \in Q D_{n}$ has a fundamental quotient.

Proof. By definition, $X$ has a quotient $\prod_{i=1}^{n} X_{i}$ with $X_{i}$ quotient indecomposable for every $i$. If $X_{i}$ and $X_{j}$ are not quotient incomparable, then passing to further quotients, we can suppose that they are isomorphic. If $X_{i}$ and $X_{j}$ are quotient incomparable, then so are their quotients. By an iterative process of passing to further quotients we get a product with factors either isomorphic or quotient incomparable. Applying suitable isomorphisms in the factors we get a fundamental $Q D_{n}$ space.

For the convenience of the reader we recall some basic facts about product spaces. 
Lemma 4.4. Let $M$ and $M_{i}, i=1, \ldots, n$, be subspaces of $X$. Let $q_{i}$ : $X \rightarrow X / M_{i}$ be the quotient maps and $q=q_{1} \times \ldots \times q_{n}$. Then

(a) The map $q$ is surjective if and only if $M_{1}^{\perp}+\ldots+M_{n}^{\perp}$ is a direct sum.

(b) Suppose that $q$ is surjective and that $M+M_{i}$ is infinite-codimensional for every $i$. Then there exist infinite-codimensional subspaces $L_{i} \supseteq M_{i}$ and a surjective map from $X / M$ onto $\prod_{i=1}^{n}\left(X / L_{i}\right)$.

Proof. (a) Observe that, via the natural identifications $\left(X / M_{i}\right)^{*}=M_{i}^{\perp}$, the dual map $q^{*}: M_{1}^{\perp} \times \ldots \times M_{n}^{\perp} \rightarrow X^{*}$ is given by $q^{*}\left(f_{1}, \ldots, f_{n}\right)=f_{1}+$ $\ldots+f_{n}$. Now, $q$ is surjective if and only if $q^{*}$ is injective with closed range, and this amounts to $M_{1}^{\perp}+\ldots+M_{n}^{\perp}$ being a direct sum.

(b) Since $q$ : $X \rightarrow \prod_{i=1}^{n} X / M_{i}$ is surjective, $M_{1}^{\perp}+\ldots+M_{n}^{\perp}$ is a direct sum. Thus there exists $0<c<1$ such that for every $f_{i} \in M_{i}^{\perp}, i=1, \ldots, n$, we have $\left\|\sum_{i=1}^{n} f_{i}\right\| \geq c \sum_{i=1}^{n}\left\|f_{i}\right\|$.

The sums $M+M_{i}$ are infinite-codimensional. Thus by Lemma 2.4 there exist subspaces $N_{i} \supseteq M$ and $L_{i} \supseteq M_{i}$, and compact operators $K_{i}: X \rightarrow X$ with $\left\|K_{i}\right\|<c / 2$ so that $\left(I+K_{i}\right)^{*} L_{i}^{\perp}=N_{i}^{\perp}$.

Let $f_{i} \in L_{i}^{\perp}, i=1, \ldots, n$. Then

$$
\left\|\sum_{i=1}^{n}\left(I+K_{i}^{*}\right)\left(f_{i}\right)\right\| \geq\left\|\sum_{i=1}^{n} f_{i}\right\|-\left\|\sum_{i=1}^{n} K_{i}^{*}\left(f_{i}\right)\right\| \geq(c / 2) \sum_{i=1}^{n}\left\|f_{i}\right\| .
$$

Thus the subspaces $N_{i}^{\perp}$ form a direct sum. Hence $p: X \rightarrow \prod_{i=1}^{n}\left(X / N_{i}\right)$ is onto, by (a). As $N_{i}^{\perp} \subseteq M^{\perp}, p$ admits a factorization $p=p_{0} Q_{X / M}$ through $X / M$. Since $I+K_{i}$ induces an isomorphism $U_{i}$ from $X / N_{i}$ onto $X / L_{i}$ and $p$ is surjective, composing $p_{0}$ and $U_{1} \times \ldots \times U_{n}$ we get a surjective map from $X / M$ onto $\prod_{i=1}^{n}\left(X / L_{i}\right)$.

Proposition 4.5. Let $X \in Q D_{n}$ and $Y \in Q \underset{\widetilde{X}}{D_{m}}$. Suppose that $Y$ is isomorphic to a quotient of $X$ (so that $m \leq n)$. Let $\widetilde{X}$ and $\widetilde{Y}$ be fundamental quotients of $X$ and $Y$, respectively. Then $Y$ has a fundamental quotient $\widetilde{Z}$ such that $\widetilde{Z} \leq \widetilde{Y}$ and there exists a surjective operator from $\widetilde{X}$ onto $\widetilde{Z}$ determined by a diagonal surjective matrix.

Proof. Set $\tilde{X} \simeq \prod_{j=1}^{n} X_{j}$ and $\tilde{Y} \simeq \prod_{i=1}^{m} Y_{i}$. All spaces in the statement are isomorphic to quotients of $X$, so we can write $\tilde{X}=X / M$ and $Y_{i} \simeq X / N_{i} \simeq Y / L_{i}$ for suitable subspaces. Since $\widetilde{X}=X / M$ is quasimaximal, $M+N_{i}$ is infinite-codimensional for every $i$. So we can apply Lemma 4.4 to the onto operator $X \rightarrow \prod_{i=1}^{m} X / N_{i}$, and find a surjection $X / M \rightarrow \prod_{i=1}^{m}\left(X / V_{i}\right)$ for some $V_{i} \supseteq N_{i}$. Now $X / V_{i} \simeq Y / W_{i}=Z_{i}$ for some $W_{i} \supseteq L_{i}$, and we have a surjective operator $X / M \rightarrow \prod_{i=1}^{m} Z_{i}$. The surjective operator $\Lambda: Y \rightarrow \prod_{i=1}^{m} Y_{i} \rightarrow \prod_{i=1}^{m} Z_{i}$ factorizes through an isomorphism $U: \widetilde{Z}=Y / \operatorname{Ker} \Lambda \simeq \prod_{i=1}^{m} Z_{i}$ so that $(\widetilde{Z}, U)$ is a fundamental quotient of $Y$ 
and $\widetilde{Z} \leq \tilde{Y}$. The map $q: \widetilde{X}=X / M \rightarrow \prod_{i=1}^{m} Z_{i} \simeq \widetilde{Z}$ is surjective and it is enough to apply Proposition 3.4.

Corollary 4.6. Let $X \in Q D_{n}$. Then the relation $\leq$ defines a filter on the set of all fundamental quotients of $X$; i.e., given two fundamental quotients $\widetilde{X}_{1}, \widetilde{X}_{2}$, there exists a fundamental quotient $\widetilde{X}_{3}$ such that $\widetilde{X}_{3} \leq \widetilde{X}_{1}$ and $\widetilde{X}_{3} \leq \widetilde{X}_{2}$.

Proof. Taking $Y=X, \widetilde{X}=\widetilde{X}_{1}$ and $\widetilde{Y}=\widetilde{X}_{2}$ in Proposition 4.5, we get a fundamental quotient $\widetilde{X}_{3} \leq \widetilde{X}_{2}$ and a diagonal surjective matrix from $\widetilde{X}_{1}$ onto $\widetilde{X}_{3}$. Thus $\widetilde{X}_{3} \leq \widetilde{X}_{1}$.

For a fundamental quotient $\tilde{X} \simeq \prod_{j=1}^{n} X_{j}$ we set $\chi(\widetilde{X})=\chi\left(\prod_{j=1}^{n} X_{j}\right)$.

COrollary 4.7. Let $X$ be a $Q D_{n}$ space and let $\widetilde{X}_{1}$ and $\widetilde{X}_{2}$ be fundamental quotients of $X$. Then $\chi\left(\widetilde{X}_{1}\right)=\chi\left(\widetilde{X}_{2}\right)$.

Proof. By Corollary 4.6, it is enough to consider the case $\widetilde{X}_{1} \leq \widetilde{X}_{2}$. After a permutation of the factors, we can suppose that there is a diagonal surjective matrix from $\widetilde{X}_{2} \simeq \prod_{j=1}^{n} X_{j}$ into $\widetilde{X}_{1} \simeq \prod_{j=1}^{n} Y_{j}$. Then, by (2), $X_{i}=X_{j}$ if and only if $Y_{i}=Y_{j}$, which yields the assertion.

TheOREM 4.8. Let $X \in Q D_{n}$ and $Y \in Q D_{m}$ be complex spaces. Suppose that $Y$ is isomorphic to a quotient of $X$. Let $\widetilde{X} \simeq \prod_{j=1}^{n} X_{j}$ and $\widetilde{Y} \simeq \prod_{i=1}^{m} Y_{i}$ be fundamental quotients of $X$ and $Y$, respectively, so that there exists a surjective operator from $\widetilde{X}$ onto $\widetilde{Y}$ with matrix $(D \mid 0)$, where $D$ is a diagonal matrix. Then the map

$$
\psi: M_{\widetilde{X}, \tilde{Y}}(\mathbb{C}) \rightarrow L(X, \tilde{Y}) / S C(X, \widetilde{Y})
$$

defined by $\psi(A)=\left[D A Q_{\tilde{X}}\right]$ is bijective. In particular,

$$
\operatorname{dim} L(X, Y) / S C(X, Y) \leq \operatorname{dim} M_{\tilde{X}, \tilde{Y}} .
$$

Proof. Let $T \in L(X, Y)$. Since $\widetilde{X}$ is quasi-maximal, we can apply Proposition 2.8(b) with $Y=Y_{i}$. Then we obtain quotients $Q_{Z_{i}}: Y_{i} \rightarrow Z_{i}$ and operators $S_{i}: \widetilde{X} \rightarrow Z_{i}$ such that $S_{i} Q_{\widetilde{X}}-Q_{Z_{i}} T \in S C\left(X, Z_{i}\right)$. Passing to further quotients of $Z_{i}$, we can suppose that $\prod_{i=1}^{m} Z_{i}$ is a fundamental $Q D_{m}$ space. Let $\widetilde{Z} \simeq \prod_{i=1}^{m} Z_{i}$ be the corresponding fundamental quotient. Taking $S=\prod_{j=1}^{m} S_{j}$ and $Q_{\widetilde{Z}} \in L(\widetilde{Y}, \widetilde{Z})$, where $Q_{\widetilde{Z}}\left(y_{1}, \ldots, y_{m}\right)=$ $\left(Q_{Z_{1}}\left(y_{1}\right), \ldots, Q_{Z_{m}}\left(y_{m}\right)\right)$, we obtain

$$
S Q_{\widetilde{X}}-Q_{\widetilde{Z}} T \in S C(X, \widetilde{Z})
$$

Applying Proposition 3.8 to $\widetilde{X}, \widetilde{Z}$ and $D_{1}=Q_{\widetilde{Z}} D$, we have $S=D_{1} A+$ $K_{1}$ with $A \in M_{\widetilde{X}, \widetilde{Y}}(\mathbb{C})$ and $K_{1} \in S C(\widetilde{X}, \widetilde{Z})$. Putting this in (3), we get $D_{1} A Q_{\widetilde{X}}-Q_{\widetilde{Z}} T=Q_{\widetilde{Z}}\left(D A Q_{\widetilde{X}}-T\right) \in S C(X, \widetilde{Z})$. As $\widetilde{Z}$ is quasi-maximal, $D A Q_{\tilde{X}}-T \in S C(X, \widetilde{Y})$ by Proposition 2.8 and $\psi$ is onto. 
Now, if $D A Q_{\widetilde{X}} \in S C(X, \widetilde{Y})$, then $D A \in S C(\widetilde{X}, \widetilde{Y})$, i.e., $\phi_{i} a_{i j}$ is strictly cosingular for every $i, j$. As every $\phi_{i}$ is onto, it follows that $a_{i j}=0$ for every $i, j$ and $A=0$, so $\psi$ is injective. Hence

$$
\operatorname{dim} L(X, Y) / S C(X, Y) \leq \operatorname{dim} L(X, \tilde{Y}) / S C(X, \widetilde{Y})=\operatorname{dim} M_{\widetilde{X}, \widetilde{Y}}(\mathbb{C}),
$$

by Proposition 3.8 .

Remark 4.9. Given complex spaces $X \in Q D_{n}$ and $Y \in Q D_{m}$, with $Y$ isomorphic to a quotient of $X$, Proposition 4.5 ensures the existence of fundamental quotients $\widetilde{X}, \widetilde{Y}$ under the hypothesis of Theorem 4.8 .

REMARK 4.10. The map $\psi: M_{\widetilde{X}, \tilde{Y}}(\mathbb{C}) \rightarrow L(X, \tilde{Y}) / S C(X, \widetilde{Y})$ that appears in Theorem 4.8 depends on the choice of the fundamental quotients $\widetilde{X}$ and $\widetilde{Y}$. However, for different choices of fundamental quotients $\widetilde{X}_{1}, \widetilde{X}_{2}$ and $\widetilde{Y}_{1}, \widetilde{Y}_{2}$, the subspaces of $m \times n$ matrices $M_{\widetilde{X}_{1}, \widetilde{Y}_{1}}(\mathbb{C})$ and $M_{\widetilde{X}_{2}, \widetilde{Y}_{2}}(\mathbb{C})$ coincide up to permutation.

Denote by $\psi_{\widetilde{X}}$ the isomorphism $\psi$ of Theorem 4.8 when $X=Y, \widetilde{X}=\widetilde{Y}$ and $D=I$.

Theorem 4.11. Let $X \in Q D_{n}$ be a complex space. Let $\widetilde{X} \simeq \prod_{j=1}^{n} X_{j}$ be a fundamental quotient of $X$. Then the map

$$
\Theta: L(X) / S C(X) \rightarrow M_{\tilde{X}, \tilde{X}}
$$

defined by $\Theta[T]=\psi_{\tilde{X}}^{-1}\left[Q_{\widetilde{X}} T\right]$ is an algebra isomorphism from $L(X) / S C(X)$ onto a subalgebra of $M_{\widetilde{X}, \widetilde{X}}$.

Proof. First of all, $\Theta$ is injective by Proposition 2.8(a) and Theorem 4.8.

In view of Theorem 4.8 , it just remains to prove the multiplicativity.

Let $\left[T_{1}\right],\left[T_{2}\right] \in L(X) / S C(X)$ with $\Theta\left[T_{1}\right]=A_{1}$ and $\Theta\left[T_{2}\right]=A_{2}$. Then $Q_{\widetilde{X}} T_{1}-A_{1} Q_{\widetilde{X}}=S_{1}$ and $Q_{\widetilde{X}} T_{2}-A_{2} Q_{\widetilde{X}}=S_{2}$ with $S_{1}, S_{2} \in S C(X, \widetilde{X})$. Thus

$$
Q_{\widetilde{X}} T_{1} T_{2}-A_{1} A_{2} Q_{\widetilde{X}}=A_{1} S_{2}+S_{1} T_{2} \in S C(X, \widetilde{X})
$$

and $\Theta\left(\left[T_{1}\right]\left[T_{2}\right]\right)=\Theta\left(\left[T_{1} T_{2}\right]\right)=A_{1} A_{2}=\Theta\left[T_{1}\right] \Theta\left[T_{2}\right]$.

5. Spectral theory on $Q D_{n}$ spaces. Recall that an operator $T \in$ $L(X, Y)$ is said to be semi-Fredholm if $R(T)$ is closed and either $\operatorname{Ker}(T)$ or $\operatorname{dim} Y / R(T)$ is finite. For a semi-Fredholm operator $T$ we define the index by

$$
\operatorname{ind}(T):=\operatorname{dim} \operatorname{Ker}(T)-\operatorname{dim} Y / R(T) \in \mathbb{Z} \cup\{ \pm \infty\} .
$$

It is well known that the index is a continuous map [10, Theorem IV.5.17].

The operator $T \in L(X, Y)$ is said to be Fredholm if it is semi-Fredholm with finite index. The essential spectrum of $T \in L(X)$ is defined as follows:

$$
\sigma_{\mathrm{e}}(T)=\{\lambda: \lambda I-T \text { is not Fredholm }\} .
$$


The following result follows from the fact that the strictly cosingular operators $S C$ form a perturbation ideal in the sense of Heuser [9, Section $51]$.

Proposition 5.1. An operator $T \in L(X)$ is Fredholm if and only if $[T]$ is invertible in $L(X) / S C(X)$. In particular, $\sigma_{\mathrm{e}}(T)=\sigma([T])$ for every $T \in L(X)$.

Proposition 5.2. Let $X$ be a $Q D_{n}$ space. Then $\operatorname{ind}(T)=0$ for every semi-Fredholm operator $T$ on $X$.

Proof. We suppose first that $X$ is complex. In this case it is enough to observe that ind $(T-\lambda I)$ is a continuous discrete function in $\lambda$, that $\mathbb{C} \backslash \sigma_{\mathrm{e}}(T)$ is connected by Proposition 5.1 and Theorem 4.11, and that ind $(T-\lambda I)=0$ for $|\lambda|>\|T\|$.

Suppose now that $X$ is a real space and that $X_{\mathbb{C}}$ denotes its complexification. Then $X_{\mathbb{C}}$ is isomorphic to $X \oplus X$ as a real space, thus it is a real $Q D_{2 n}$ space [6, Theorem 2]. As every complex quotient of $X_{\mathbb{C}}$ is also a real quotient, $X_{\mathbb{C}}$ is a complex $Q D_{m}$ space for some $m \leq 2 n$. If $T_{\mathbb{C}}$ is the complexification of a semi-Fredholm operator $T$, then $T_{\mathbb{C}}$ is also semi-Fredholm and $\operatorname{ind}\left(T_{\mathbb{C}}\right)=\operatorname{ind}(T)$. By the first part $\operatorname{ind}\left(T_{\mathbb{C}}\right)=0$, so ind $(T)=0$.

Corollary 5.3. Suppose that $X$ is a $Q D_{n}$ space for some $n$. Then $X$ is isomorphic neither to any of its proper subspaces nor to any of its proper quotients.

Proof. Let $Q: X \rightarrow Y$ be a quotient and let $U: Y \rightarrow X$ be an isomorphism. Then $U Q$ is a semi-Fredholm operator on $X$. By Proposition 5.2, $U Q$ is Fredholm of index 0 . As $U Q$ is surjective, $\operatorname{Ker} Q=0$ and $Q=I$. The proof for the case of subspaces is analogous.

The following results are a consequence of Theorem 4.11.

Proposition 5.4. Let $X$ be a complex $Q D_{n}$ space and let $T \in L(X)$. Then

(a) $\left|\sigma_{\mathrm{e}}(T)\right| \leq n$.

(b) $T$ is not Fredholm if and only if there exists a non-strictly cosingular operator $U \in L(X)$ such that $T U \in S C(X)$.

Proof. (a) It is enough to observe that $\sigma_{\mathrm{e}}(T)$ coincides with $\sigma([T])$ by Proposition 5.1, which is the set of eigenvalues of the scalar matrix $\Theta[T]$ by Theorem 4.11.

(b) If $T$ is Fredholm, then $[T] \in L(X) / S C(X)$ is invertible, and $T U \in$ $S C(X)$ implies $U \in S C(X)$.

Conversely, suppose that $T$ is not Fredholm and $[T] \neq 0$. Let $p$ be the minimal polynomial of $[T]$. Since $[T]$ is not invertible, it follows that $p(0)=0$ and there exists a polynomial $q$ with $\operatorname{deg} q=\operatorname{deg} p-1$ such that $T q(T)=$ 
$p(T)$. By the definition of a minimal polynomial, $U=q(T)$ cannot be strictly cosingular. Since $T U=p(T) \in S C(X)$, the result is proved.

For a complex $Q D_{n}$ space $X$, Theorem 4.11 allows us to identify the Calkin algebra $L(X) / S C(X)$ with a subalgebra of $M_{\widetilde{X}, \tilde{X}}$. The next result provides additional information on such subalgebras.

Theorem 5.5. Let $n \in \mathbb{N}$. A complex space $X$ is $n$-decomposable if and only if there exists an operator $T \in L(X)$ such that $\left|\sigma_{\mathrm{e}}(T)\right|=n$.

Proof. Suppose that $X=X_{1} \oplus \ldots \oplus X_{n}$. Then

$$
T\left(x_{1}, x_{2}, \ldots, x_{n}\right):=\left(x_{1}, 2 x_{2}, \ldots, n x_{n}\right)
$$

defines an operator on $T \in L(X)$ such that $\sigma_{\mathrm{e}}(T)=\{1,2, \ldots, n\}$.

Conversely, let $T \in L(X)$ be such that $\sigma_{\mathrm{e}}(T)=\left\{\lambda_{1}, \ldots, \lambda_{n}\right\}$ with $\lambda_{i} \neq \lambda_{j}$ for $i \neq j$. By [4, Theorem V.1.8], $\lambda I-T$ is invertible on $\mathbb{C} \backslash\left\{\lambda_{1}, \ldots, \lambda_{n}\right\}$ with the possible exceptions of isolated points.

We select closed simple curves $C_{1}, \ldots, C_{n}$ on $\mathbb{C} \backslash \sigma(T)$ which do not intersect so that each $\lambda_{i}$ is in the interior of $C_{i}$ and every point in $\sigma(T)$ is contained in the interior of $C_{j}$ for some $j$.

The analytic operational calculus [12, Section V.8] allows us to define

$$
P_{i}:=\int_{C_{i}}(\lambda I-T)^{-1} d \lambda, \quad i=1, \ldots, n .
$$

Then each $P_{i}$ is a projection and $X=R\left(P_{1}\right) \oplus \ldots \oplus R\left(P_{n}\right)$ [12, Theorem V.9.1]. Thus, $X$ is $n$-decomposable.

REMARK 5.6. Clearly, the proof of Theorem 5.5 shows that a complex Banach space is $n$-decomposable if and only if there exists an operator $T \in$ $L(X)$ such that $\sigma_{\mathrm{e}}(T)$ has $n$ components, i.e., $\sigma_{\mathrm{e}}(T)$ admits a partition into $n$ non-empty compact subsets.

The following example shows that in Proposition 5.4 it is not enough to suppose $X$ indecomposable in order to get $\left|\sigma_{\mathrm{e}}(T)\right|=1$ for every $T \in L(X)$.

Example $[8,(4.2)]$. There exists a complex indecomposable space $X$ and an operator $S \in L(X)$ such that $\sigma_{\mathrm{e}}(S)=\{\lambda \in \mathbb{C}:|\lambda|=1\}$. Moreover $\operatorname{ind}(S)=-1$.

An interesting question is to describe the subalgebras of $M_{n}(\mathbb{C})$ which can be identified with the Calkin algebra $L(X) / S C(X)$ for some $Q D_{n}$ space $X$, as in Theorem 4.11.

In the case of an indecomposable $Q D_{n}$ space $X$, Theorem 5.5 implies that $\left|\sigma_{\mathrm{e}}(T)\right|=1$ for every $T \in L(X)$. Therefore, $L(X) / S C(X)$ can be identified by Theorem 4.11 with a subalgebra $\mathcal{A}$ of $M_{n}(\mathbb{C})$ such that each matrix in $\mathcal{A}$ has only one eigenvalue. A trivial example of such a subalgebra is $\mathcal{A}=\{\lambda I: \lambda \in \mathbb{C}\}$. A second example is the algebra of all upper triangular 
matrices in $M_{n}(\mathbb{C})$ with constant diagonal. We denote this algebra by $\mathcal{U}_{n}$. Note that the first example is a subalgebra of $\mathcal{U}_{n}$. Let us see that the Kolchin Theorem on unipotent algebraic groups allows us to show that up to a change of basis all the subalgebras $\mathcal{A}$ are of this kind.

We recall that a matrix $A \in M_{n}(\mathbb{C})$ is said to be unipotent if its sole eigenvalue is 1, i.e. if its characteristic polynomial is $q_{A}(x)=(x-1)^{n}$.

Theorem 5.7. Let $X$ be an indecomposable $Q D_{n}$ space. Then the Calkin algebra $L(X) / S C(X)$ can be identified with a subalgebra of $\mathcal{U}_{n}$.

Proof. Let $\mathcal{A}$ be the subalgebra of $M_{n}(\mathbb{C})$ identified with $L(X) / S C(X)$ by Theorem 4.11 and let $G$ be the invertible elements in $\mathcal{A}$. We claim that the set $G_{\mathrm{u}}$ of all unipotent elements of $G$ is a subgroup.

Each matrix $A \in \mathcal{A}$ has a unique eigenvalue, which we denote by $\alpha(A)$. Thus, $q_{A}(x)=(x-\alpha(A))^{n}$ is the characteristic polynomial of $A$ and it is easy to see that

$$
\alpha(A)=\frac{1}{n} \operatorname{trace}(A), \quad \alpha(A)^{n}=\operatorname{det}(A) .
$$

Therefore the assignment $A \mapsto \alpha(A)$ defines a continuous map $\alpha$ from $G$ into $\mathbb{C}$ satisfying $\left(\alpha(A) \alpha(B) \alpha(A B)^{-1}\right)^{n}=\operatorname{det}(A) \operatorname{det}(B) \operatorname{det}(A B)^{-1}=1$. This implies that the function $f: G \times G \rightarrow \mathbb{C}$, defined by

$$
f(A, B)=\alpha(A) \alpha(B) \alpha(A B)^{-1},
$$

takes its values in the finite set of $n$th roots of unity. The function $f$ is continuous and $G \times G$ is connected, so $f(G \times G)$ must be a point. As $f(I, I)=1$, it follows that $\alpha(A) \alpha(B)=\alpha(A B)$ for every $A, B$ in $G$, and the claim is proved.

Since $G_{\mathrm{u}}$ is a subgroup of $G$, we can apply the Kolchin Theorem [13, Theorem 8.2] to $G_{\mathrm{u}}$, and we deduce that there exists an invertible matrix $B \in M_{n}(\mathbb{C})$ such that $B^{-1} G_{\mathrm{u}} B$ is a subgroup of the group $U_{n}$ of all upper triangular unipotent matrices in $M_{n}(\mathbb{C})$. The algebras generated by $G_{\mathrm{u}}$ and $U_{n}$ in $M_{n}(\mathbb{C})$ are $\mathcal{A}$ and $\mathcal{U}_{n}$, respectively. Thus, $B^{-1} \mathcal{A} B \subseteq \mathcal{U}_{n}$, as we wanted to prove.

\section{References}

[1] S. A. Argyros and V. Felouzis, Interpolating hereditarily indecomposable Banach spaces, J. Amer. Math. Soc. 13 (2000), 243-294.

[2] V. Ferenczi, Hereditarily finitely decomposable spaces, Studia Math. 123 (1997), 135-149.

[3] -, Quotient hereditarily indecomposable Banach spaces, Canad. J. Math. 51 (1999), 566-584.

[4] S. Goldberg, Unbounded Linear Operators, McGraw-Hill, 1966. 
[5] M. González and J. M. Herrera, Operators on quotient indecomposable spaces, Publ. Math. Debrecen 59 (2001), 271-288.

[6] - - - Finitely decomposable Banach spaces and the three-space property, Arch. Math. (Basel), to appear.

[7] W. T. Gowers and B. Maurey, The unconditional basic sequence problem, J. Amer. Math. Soc. 6 (1993), 851-874.

[8] —, - Banach spaces with small spaces of operators, Math. Ann. 307 (1997), 543568.

[9] H. G. Heuser, Functional Analysis, Wiley, 1982.

[10] T. Kato, Perturbation Theory for Linear Operators, Springer, 1976.

[11] D. Przeworska-Rolewicz and S. Rolewicz, Equations in Linear Spaces, PWN-Polish Sci. Publ., 1968.

[12] A. E. Taylor and D. C. Lay, Introduction to Functional Analysis, Wiley, 1980.

[13] W. C. Waterhouse, Introduction to Affine Group Schemes, Springer, 1979.

Departamento de Matemáticas

Universidad de Cantabria

E-39071 Santander, Spain

E-mail: gonzalem@unican.es

herreraj@unican.es

Received March 4, 2002

Revised version February 17, 2003 